\title{
Vibrations of Micro-eV Energies in Nanocrystalline Microstructures
}

\author{
A. F. Yue, A. B. Papandrew, O. Delaire, and B. Fultz \\ Division of Engineering and Applied Science, California Institute of Technology, Pasadena, California 91125, USA \\ Z. Chowdhuri, ${ }^{*}$ R. M. Dimeo, and D. A. Neumann \\ NIST Center for Neutron Research, National Institute of Standards and Technology, Gaithersburg, Maryland 20899, USA
}

(Received 27 April 2004; published 10 November 2004)

\begin{abstract}
The phonon density of states of nanocrystalline bcc Fe and nanocrystalline fcc $\mathrm{Ni}_{3} \mathrm{Fe}$ were measured by inelastic neutron scattering in two different ranges of energy. As has been reported previously, the nanocrystalline materials showed enhancements in their phonon density of states at energies from 2 to $15 \mathrm{meV}$, compared to control samples composed of large crystals. The present measurements were extended to energies in the micro-eV range, and showed significant, but smaller, enhancements in the number of modes in the energy range from 5 to $18 \mu \mathrm{eV}$. These modes of micro-eV energies provide a long-wavelength limit that bounds the fraction of modes at milli-eV energies originating with the cooperative dynamics of the nanocrystalline microstructure.
\end{abstract}

DOI: $10.1103 /$ PhysRevLett.93.205501

PACS numbers: 63.22.+m, 61.46.+w, 81.07.-b

The vibrational spectra of nanocrystalline materials differ from those of materials composed of larger crystals. Earlier measurements of Debye-Waller factors and Lamb-Mössbauer factors showed large mean-squared atomic displacements in nanocrystalline materials [14]. More recently, neutron and $x$-ray inelastic scattering measurements revealed that nanocrystalline materials have an increased number of vibrational modes at the highest and lowest energies of their measured spectra, compared to materials with crystals of conventional sizes [5-12]. Enhanced intensity above the high-energy cutoff of the bulk material has been attributed to phonon lifetime broadening caused by phonon interactions with grain boundaries [9-11] and recent measurements on iron have isolated a contribution from surface oxides [12]. The increased number of phonon modes at low energies [5-11] is less well understood. The phonon density of states (DOS) at low energies is up to a factor of 5 times larger than the DOS of control samples of material composed of larger crystals [10]. The number of these low-energy modes was found to increase with the inverse of the crystallite size, implying a scaling with the number of grain boundaries. Surface vibrational modes could be responsible for such behavior because grain boundaries have elastic constants differing from the crystal interiors. There are some experimental and computational reports that the low-energy phonon DOS in nanocrystalline materials scales linearly with energy, indicative of twodimensional vibrations [13,14]. Other theoretical models include the idea of a nonintegral spatial dimension of the low-energy modes [15-18].

Surface modes on individual, isolated crystallites have a maximum wavelength comparable to the crystallite size, and therefore a lower bound on their energy. For a wave velocity of $2 \mathrm{~km} / \mathrm{s}$ and a particle of $10 \mathrm{~nm}$ dimension, the characteristic energy is $1 \mathrm{meV}$. Energies of a milli-electron volt have been approximately the lower limit of the inelastic spectra measured to date. Surface modes around nanoparticles should not extend to energies much below $100 \mu \mathrm{eV}$, however. The present experiment was designed to measure the phonon DOS of interconnected nanocrystals at much lower energies. We report significant enhancements in the phonon DOS of nanocrystalline materials at the extremely low energies from 3-18 $\mu \mathrm{eV}$. These modes must involve long-wavelength cooperative motions over distances of many nanoparticles. A comparison of the dynamics in the micro-eV and milli-eV energy regimes offers a more detailed picture of the dynamics within and around individual nanoparticles, and shows similarities to the dynamics of other disordered solids.

Nanocrystalline Fe powders were synthesized by mechanical attrition in a Union Process 01-HD mixer/mill. Approximately $100 \mathrm{~g}$ of Fe powder of $99.9 \%$ purity were placed in hardened steel vials with steel balls in an argon atmosphere, and were milled for $36 \mathrm{~h}$ with a ball-topowder weight ratio of 20:1. A similar method was used to produce the nanocrystalline $\mathrm{Ni}_{3} \mathrm{Fe}$ in a Spex $8000 \mathrm{mixer} / \mathrm{mill}$. Samples of $\mathrm{Ni}_{3} \mathrm{Fe}$ and $\mathrm{Fe}$ prepared in this way are composed of nanocrystals in mutual contact through grain boundaries $[8,10]$. Control samples with larger crystals were prepared by annealing some of the milled powder at $550^{\circ} \mathrm{C}$. X-ray diffraction line shapes, analyzed by a $\Delta Q$ vs $Q$ method, showed that the average crystallite size was $10 \mathrm{~nm}$ for the nanocrystalline $\mathrm{Fe}$, $6 \mathrm{~nm}$ for the nanocrystalline $\mathrm{Ni}_{3} \mathrm{Fe}$, and 35 and $50 \mathrm{~nm}$ for the control samples of $\mathrm{Fe}$ and $\mathrm{Ni}_{3} \mathrm{Fe}$, respectively, (see also Ref. [9]). Prompt-gamma activation analyses of hydrogen concentrations were performed at the NIST Center for Neutron Research on both nanocrystalline and control samples. The sample of nanocrystalline Fe contained $0.19 \pm 0.02$ at. $\% \mathrm{H}$, similar to the control 
sample of Fe with $0.16 \pm 0.02$, and the nanocrystalline $\mathrm{Ni}_{3} \mathrm{Fe}$ had a hydrogen content of 0.4 at. \% $\mathrm{H}$. It is unclear, however, if this hydrogen was present as surface hydrocarbons, especially for the $\mathrm{Ni}_{3} \mathrm{Fe}$ sample for which an organic surfactant was used in preparation, or if the hydrogen was within the material.

For inelastic neutron scattering spectra of $\mathrm{Fe}$ at $\mathrm{meV}$ energies, samples of nanocrystalline powder and samples of the control powder were measured on the Pharos spectrometer at the Los Alamos Neutron Science Center at the Los Alamos National Laboratory, and on the LRMECS spectrometer at the Intense Pulsed Neutron Source at the Argonne National Laboratory. The energies of the incident neutrons were 50 and $60 \mathrm{meV}$, respectively. Similar measurements with incident neutrons of $45 \mathrm{meV}$ energy were performed on samples of $\mathrm{Ni}_{3} \mathrm{Fe}$ with the LRMECS spectrometer. The results were essentially the same as those from prior measurements with a triple-axis instrument $[7,10]$, although a better sampling of phonons at all $Q$ is possible with time-of-flight instruments ( $\hbar Q$ is the momentum transfer).

Inelastic neutron scattering measurements at micro-eV energy transfers were performed with the high-flux backscattering spectrometer, HFBS, on NG-2 at the NIST Center for Neutron Research. The spectrometer was operated with a fixed final energy of $2.08 \mathrm{meV}$. The incident energy was varied with a $\mathrm{Si}(111)$ monochromator mounted on a Doppler drive. Samples of nanocrystalline powder and control samples, each about $20 \mathrm{~g}$, were placed in thin-walled annular aluminum cans mounted on a closed-cycle refrigerator in the spectrometer. Data were obtained with energy transfer $\varepsilon$ in the range of $\pm 18 \mu \mathrm{eV}$ and with the samples at temperatures of $50 \mathrm{~K}$ and $293 \mathrm{~K}$. The detectors provided 16 values of $Q$ from 0.25 to $1.75 \AA^{-1}$. The spectrometer had an energy resolution (FWHM) of $0.95 \mu \mathrm{eV}$, as measured with a vanadium standard. Spectra were acquired with the samples at am- bient temperature and at a cryogenic temperature. A total of 12 pairs of spectra were acquired from the nanocrystalline and control samples of $\mathrm{Fe}$, and 14 from the $\mathrm{Ni}_{3} \mathrm{Fe}$.

Figure 1(a) presents scattered neutron counts from $\mathrm{Fe}$ at micro-eV energies measured with the HFBS instrument, after summing over the full range of accessible angles and after subtracting the background measurement from the empty can. Figure 1(c) presents similar data at milli$\mathrm{eV}$ energies from the Pharos and LRMECS instruments, again at $293 \mathrm{~K}$. Corresponding data for the $\mathrm{Ni}_{3} \mathrm{Fe}$ samples are shown Fig. 2(a) and Fig. 2(c). The Pharos and LRMECS data were corrected for multiphonon scattering through an iterative procedure described previously [19]. The HFBS data sets for $\mathrm{Fe}$ and $\mathrm{Ni}_{3} \mathrm{Fe}$ were corrected for background only, as multiphonon scattering is not significant at low values of $Q$. The DAVE software package was used for reducing the backscattering data [20].

Figs. 1(b) and 2(b) present HFBS data measured at cryogenic temperatures on the $\mathrm{Fe}$ and $\mathrm{Ni}_{3} \mathrm{Fe}$ samples, respectively. Their lower intensities compared to the spectra at ambient temperature of Figs. 1(a) and 2(a) are in the ratio of the temperatures of measurement, as expected for phonon scattering at low energies. Nevertheless, the intensity of the inelastic scattering in the $\mu \mathrm{eV}$ energy range is larger for the nanocrystalline samples than the control samples at cryogenic temperature, much as for the spectra measured at ambient temperature. This also indicates that the enhanced scattering from the nanocrystalline materials is primarily an effect of phonon scattering, as opposed to quasielastic scattering that should be suppressed at cryogenic temperatures.

The experimental data show that the scattering intensity of the nanocrystalline $\mathrm{Fe}$ is enhanced by a factor of $1.2 \pm 0.05$ over that of the control sample in the $\mu \mathrm{eV}$ energy range. In the meV energy range of the Pharos and LRMECS instruments, an enhancement by a factor of $1.30 \pm 0.05$ is observed, consistent with previous mea-
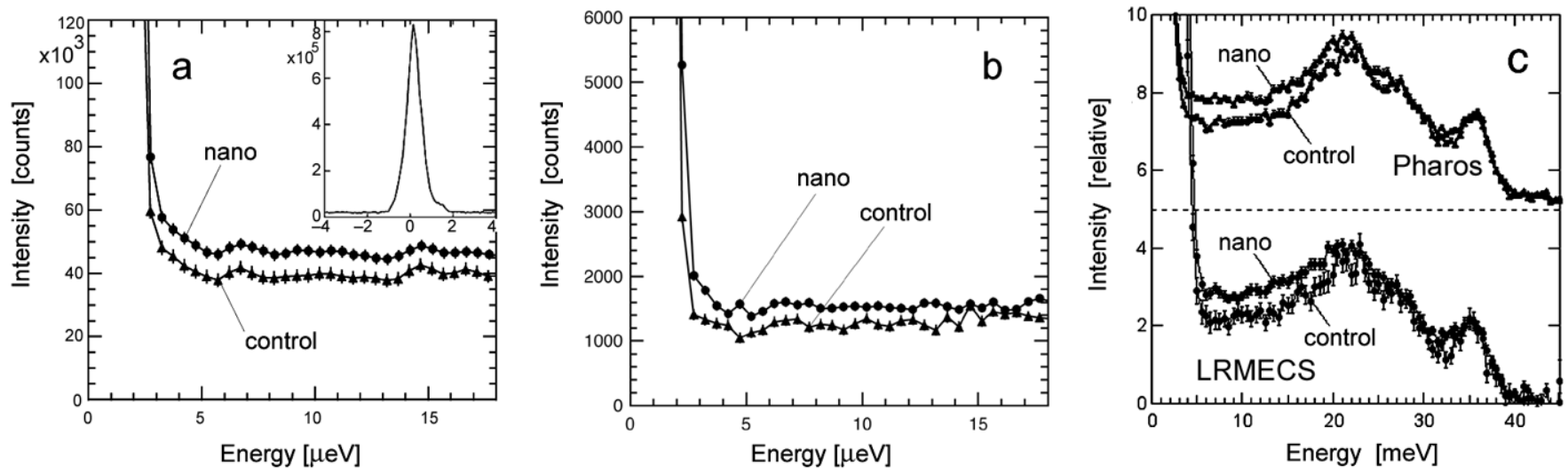

FIG. 1. Typical neutron energy loss spectra for the nanocrystalline and control samples of Fe. (a) HFBS data in the $\mu$ eV range, measured at $293 \mathrm{~K}$. The ripples in these data are an instrumental artifact caused by normalization to an inhomogeneous incident flux. Inset shows elastic peak of nanocrystalline sample. (b) HFBS data in the $\mu \mathrm{eV}$ range, measured at $10 \mathrm{~K}$. (c) Pharos and LRMECS data in the meV range, measured at $293 \mathrm{~K}$. 

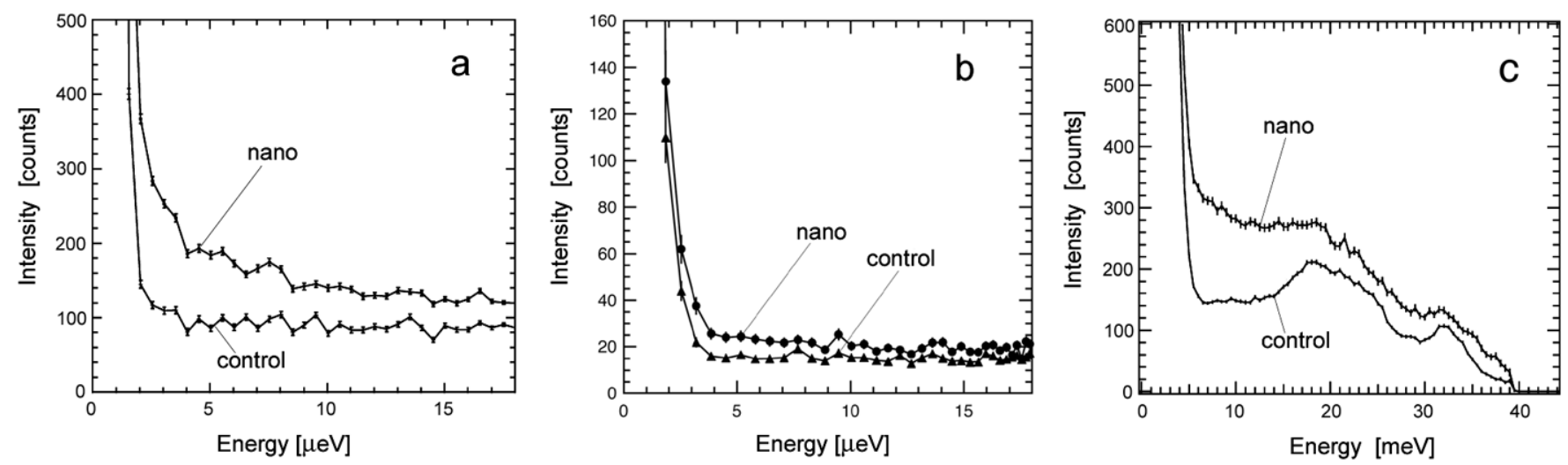

FIG. 2. Neutron energy loss spectra for the nanocrystalline and control samples of $\mathrm{Ni}_{3} \mathrm{Fe}$. (a) $\mathrm{HFBS}$ data in the $\mu \mathrm{eV}$ range, measured at $293 \mathrm{~K}$. (b) HFBS data in the $\mu \mathrm{eV}$ range, measured at $50 \mathrm{~K}$. (c) LRMECS data in the meV range, measured at $293 \mathrm{~K}$.

surements on Fe with $12 \mathrm{~nm}$ nanocrystals [8]. Such an enhancement is also present in the $\mathrm{Ni}_{3} \mathrm{Fe}$ data, but a spectral component with broad tails, centered around $\varepsilon=$ 0 , is observed in the spectrum of the nanocrystalline $\mathrm{Ni}_{3} \mathrm{Fe}$ at $293 \mathrm{~K}$ in the HFBS data. This component likely originates with quasielastic scattering from a trace amount of hydrogen within the nanocrystalline sample. The spectra measured at $50 \mathrm{~K}$, where hydrogen motions are expected to be frozen out, show less of this possible quasielastic scattering and have a flat energy spectrum beyond $10 \mu \mathrm{eV}$. Avoiding this quasielastic scattering, we find that the enhancement of modes in the nanocrystals of $\mathrm{Ni}_{3} \mathrm{Fe}$ is similar at 293 and $50 \mathrm{~K}$ for energies above approximately $10 \mu \mathrm{eV}$, consistent with phonon scattering. For $\mathrm{Ni}_{3} \mathrm{Fe}$ the enhancement in the nanocrystalline phonon DOS is a factor of $1.40 \pm 0.1$ in the $\mu \mathrm{eV}$ energy range and a factor of $1.9 \pm 0.15$ in the meV energy range.

For vibrational excitations in three dimensions, the phonon DOS in the low-energy region is expected to be proportional to the square of phonon energy, i.e., $g(\varepsilon) \propto$ $\varepsilon^{2}$. At the low momentum transfers for the HFBS spectra, the scattering is primarily one-phonon scattering. The phonon DOS, $g(\varepsilon)$, can then be obtained from the inelastic intensity $S(\varepsilon)$ as

$$
g(\varepsilon) \propto \varepsilon[1-\exp (-\varepsilon / k T)] S(\varepsilon) .
$$

By expanding the exponential for small $\varepsilon$, this equation shows that if the phonon DOS is proportional to $\varepsilon^{2}$, the measured $S(\varepsilon)$ should be constant in energy. The $S(\varepsilon)$ of Figs. 1(a), 1(b), 2(a), and 2(b) are approximately constant in energy, so the present results are largely consistent with three-dimensional vibrations. Furthermore, the similar enhancement of intensity for the nanocrystals at $\mu \mathrm{eV}$ energies and at $\mathrm{meV}$ energies suggests that the nanocrystalline DOS follows closely the characteristic $\varepsilon^{2}$ shape of the DOS of the control sample. It is not practical to compare the intensities of $S(\varepsilon)$ for spectra acquired on the different instruments, however.
With these extremely low values of $\varepsilon$ and modest $Q$ for the HFBS instrument, we expect no coherent scattering. No $Q$ dependence was found in the inelastic scattering spectra, although the intensities were larger at higher $Q$, approximately consistent with the expected $Q^{2}$ behavior. The elastic peak from the nanocrystalline sample showed a strong $Q$ dependence at small angles, consistent with small-angle neutron scattering from a nanocrystalline material.

The data of Figs. 1(c) and 2(c) show that the van Hove singularities for the longitudinal modes are at the same energies in the nanocrystalline and control samples (at 36 and $33 \mathrm{meV}$ for $\mathrm{Fe}$ and $\mathrm{Ni}_{3} \mathrm{Fe}$, respectively). Similarly, the van Hove singularities for the transverse modes, although broadened in energy, are not shifted in energy in the nanocrystalline materials (a systematic series of spectra for $\mathrm{Ni}_{3} \mathrm{Fe}$ is shown in [10]). It appears that the short-range interatomic forces for atoms within the nanocrystals are not altered significantly by the nanocrystalline microstructure.

Simplified analyses of the vibrational excitations are possible in two limiting cases. (1) If the material has homogeneous disorder and if some of the forces are soft, there should be a reduction in sound velocities and an enhancement in the number of low-energy modes. This analysis could be appropriate at $\mu \mathrm{eV}$ energies where the excitations are expected to be of long wavelength. Because the data of Figs. 1(c) and 2(c) show little effect of nanocrystallinity on the van Hove singularities, phenomena involving homogeneous disorder are less pertinent at meV energies, however. (2) If there is no coupling between the internal degrees of freedom of the different nanocrystals, the nanocrystals can be considered as independent masses connected by interfacial springs. This approach is valid only when the interfacial springs are exceptionally weak, but this is the same requirement for an enhancement of low-energy modes. Conventional crystals have low frequency modes where large effective masses are displaced against weak springs effectively 
formed by many interatomic springs in series. For a nanostructured material to have a density of independent, low-energy microstructural modes that is higher than their density for a large crystal, the interatomic forces across the grain boundaries must be smaller than the interatomic forces within the crystal by the number of interatomic springs in series across the crystal. This could be a factor of tens. A consistent set of such extremely weak interatomic forces is not expected.

Although the phonon DOS of both nanocrystalline samples were enhanced at $\mu \mathrm{eV}$ energies compared to the control sample, this enhancement was markedly greater at meV energies. The enhancement in the DOS at $\mu \mathrm{eV}$ energies is more characteristic of long waves in a homogeneous medium, indicating a suppressed velocity of sound, for example. This suggests that the extra enhancement of the DOS in nanocrystalline materials at meV energies, approximately $10 \%$ for the Fe sample and approximately $50 \%$ for the $\mathrm{Ni}_{3} \mathrm{Fe}$ sample, originates with features of the nanostructure. This change in enhancement between $\mu \mathrm{eV}$ and meV energies is the characteristic behavior of a "confinement effect," where there are no phonon modes below a long-wavelength low-energy cutoff. Micro-electron volt energies should be associated with longer-range correlation lengths. For example, for a sound velocity of $4 \mathrm{~km} / \mathrm{s}$, an energy of $10 \mu \mathrm{eV}$ corresponds to a wavelength of $0.1 \mu \mathrm{m}$. Such long distances involve cooperative motions of many nanocrystals. The compact microstructure of nanocrystals forms a coupled dynamical system, but surface modes are still expected when the grain boundaries have altered densities or force constants. The cutoff of surface modes at long wavelengths is qualitatively consistent with the smaller enhancement of the DOS at $\mu \mathrm{eV}$ energies. There also should be more surface modes in the $\mathrm{Ni}_{3} \mathrm{Fe}$ sample with its smaller crystallite size, again consistent with observations on the extra enhancement of the DOS at meV over $\mu \mathrm{eV}$ energies. We nevertheless expect such surfacerelated modes at meV energies would be coupled to the internal coordinates of the nanocrystallites. Finally we note that a relative enhancement that reaches a maximum in the meV energy range is reminiscent of the "boson peak" of glassy structures [21].

This work was supported by the U.S. Department of Energy under contract no. DE-FG02-03ER46055. The work at the NIST Center for Neutron Research is based upon activities supported by the National Science Foundation under Agreement No. DMR-0086210. Measurements at the Intense Pulsed Neutron Source were supported by the U.S. Department of Energy, BES-Materials Sciences, under Contract No. W-31-109Eng-38. This work has benefited from the use of the Los
Alamos Neutron Science Center at the Los Alamos National Laboratory, funded by the US Department of Energy under Contract No. W-7405-ENG-36. Certain commercial equipment, instruments, or materials (or suppliers, or software, ...) are identified in this paper to foster understanding. Such identification does not imply recommendation or endorsement by the National Institute of Standards and Technology, nor does it imply that the materials or equipment identified are necessarily the best available for the purpose.

*Also at Department of Materials Science and Engineering, University of Maryland, College Park, MD 20742-2115, USA

[1] G. Von Eynatten, J. Horst, K. Dransfeld, and H. E. Bömmel, Hyperfine Interact. 29, 1311 (1986).

[2] M. Hayashi, E. Gerkema, A. M. van der Kraan and I. Tamura, Phys. Rev. B 42, 9771 (1990).

[3] H. Kuwano, H. Ouyang, and B. Fultz, Nanostruct. Mater. 1, 143 (1992).

[4] L. B. Hong, C. C. Ahn and B. Fultz, J. Mater. Res. 10, 2408 (1995).

[5] B. Fultz, L. Anthony, L. J. Nagel, R. M. Nicklow, and S. Spooner, Phys. Rev. B 52, 3315 (1995).

[6] J. Trampenau, K. Bauszus, W. Petry, and U. Herr, Nanostruct. Mater. 6, 551, (1995).

[7] B. Fultz, J. L. Robertson, T. A. Stephens, L. J. Nagel, and S. Spooner, J. Appl. Phys. 79, 8318 (1996).

[8] H. N. Frase, L. J. Nagel, J. L. Robertson, and B. Fultz, Philos. Mag. B 75, 335 (1997).

[9] B. Fultz, C. C. Ahn, E. E. Alp, W. Sturhahn, T. S. Toellner, Phys. Rev. Lett. 79, 937 (1997).

[10] H. Frase, B. Fultz, and J. L. Robertson, Phys. Rev. B 57, 898 (1998).

[11] E. Bonetti, L. Pasquini, E. Sampaolesi, A. Deriu, and G. Cicognani, J. Appl. Phys. 88, 4571 (2000).

[12] L. Pasquini, A. Barla, A. I. Chumakov, O. Leupold, R. Ruffer, A. Deriu, and E. Bonetti, Phys. Rev. B 66, 073410 (2002).

[13] U. Stuhr, H. Wipf, K. H. Andersen, and H. Hahn, Phys. Rev. Lett. 81, 1449 (1998).

[14] A. Kara and T. S. Rahman, Phys. Rev. Lett. 81, 1453 (1998).

[15] J. Chadwick, J. Phys. Condens. Matter 11, 129 (1999).

[16] P. M. Derlet, R. Meyer, L. J. Lewis, U. Stuhr, and H. Van Swygenhoven, Phys. Rev. Lett. 87, 205501 (2001).

[17] D. Y. Sun, X. G. Gong, and X. Q. Wang, Phys. Rev. B 63, 193412, (2001).

[18] R. Orbach, Science 231, 814, (1986).

[19] P. D. Bogdanoff, B. Fultz and S. Rosenkranz, Phys. Rev. B 60, 3976 (1999).

[20] http://www.ncnr.nist.gov/dave

[21] W. Schirmacher, G. Diezemann, and C. Ganter, Phys. Rev. Lett. 81, 136 (1998), and references therein. 